\title{
HEAT STRESS RISK PREDICTION BY USING BAYESIAN NET MODEL WITH SENSOR NETWORK
}

\author{
Kanchan M. Taiwade ${ }^{1}$ and Prof. Prakash S. Mohod ${ }^{2}$ \\ ${ }^{1}$ Department of Computer Engineering, Nagpur University, Nagpur, India \\ kanchan.suryawanshi1111egmail.com \\ ${ }^{2}$ Head of Dept of CSE, GHRIETW, Nagpur, India \\ psmohodegmail.com
}

\begin{abstract}
With advancement in use of automation system, it is also desired to be able to know about the susceptible risk in advance for taking the preventive measures either automatically or manually. Disaster management is such an area where operatives wearing the suits and performing the activities are prone to the risk of heat stress which may cause mental impairments along with other serious effects leading to death. Such type of risk occurs in human body by not being able to compensate the heat generated into the surrounding air. The paper presents the concept of mechanism which can be used to prevent such situation by activating an alert to the operative or invoke cooling mechanism automatically before onset of the risk. The Bayesian Network Model is used to predict the onset of the risk. The model is based on the probabilities gives flexibility and simplicity in modeling the system. The system was trained with appropriate data and then compared with the real time parameters to check whether possibility of risk or not. Only those body parameters are considered which directly or indirectly participate in indicating heat stress or its onset.
\end{abstract}

\section{KEYWORDS}

Learning model, parameter selection, preventive mechanism, training data.

\section{INTRODUCTION}

The operatives of the disaster management crew for example a fire-fighting operative have to wear protective suits made up of specific material sometimes are of highly insulating material. These materials do not lead the heat to be passed outside of the suit leading to increased temperature inside the suit, also the operative performs some activities that also produces increase in the body heat, such situation can put the operative on the risk of heat stress because of the increased storage of heat, since body can not compensate it naturally because of the suit. By looking at the scenario, a system can be developed, in fact various efforts have been made in developing such system some of them were carrying an alert mechanism and some tried to accompany the fans along with the suit. The idea is to monitor the body parameters that actively participate in increasing the probability of onset of heat stress such as mean skin temperature, increased heart rate, decreased pulse rate, increased accelerations of the body; specifically limbs and arms, increased $\mathrm{CO}_{2}$ level etc. any abnormality in any of the data which can lead to heat stress, if observed, the operative will be informed by invoking an alert mechanism. The problem Natarajan Meghanathan et al. (Eds) : ICCSEA, SPPR, VLSI, WiMoA, SCAI, CNSA, WeST - 2014 pp. 49-57, 2014. (C) CS \& IT-CSCP 2014

DOI : $10.5121 /$ csit. 2014.4713 
with the alert mechanism is that even if the operative is informed by the alert mechanism, the operative may forget to actuate the preventive mechanism instead it is more desirable that the cooling system such as fan to be actuated automatically before the onset of the risk. For this purpose it is needed to monitor the body parameters on the regular time basis and if the parameter reading comes abnormal the fan is actuated automatically.

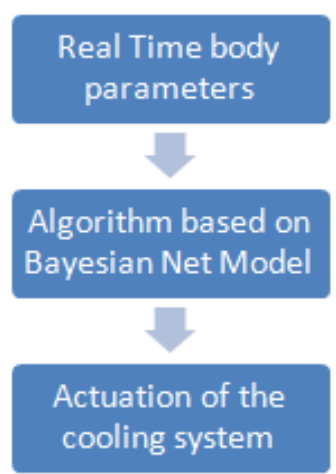

Fig1 : Execution phases

For checking if there is probability of onset of the risk or not a model need to be considered so that decision making will be based on this model. The model described in this paper is a Bayesian Net Model. Using this model for decision making by the system is quite simple as compared to other model since the Bayesian Network model does not require to have all and exact knowledge as input. The author has tried to develop such system for educational purpose.

\section{METHODOLOGY}

Since the real time parameters are to be monitored on time basis the system needed an external hardware consisting of sensors, analog-to-digital converters, relays etc to provide the real time input to the system. The basic focus is on the Bayesian algorithm with which modeling the dependencies between the parameters had been very easy because of the flexibility provided by this model. Basically the predictor was trained with the real time data; specifically the special cases; since not all the keen knowledge need to be provided. This is because the conditional dependencies can be modeled well using this model.

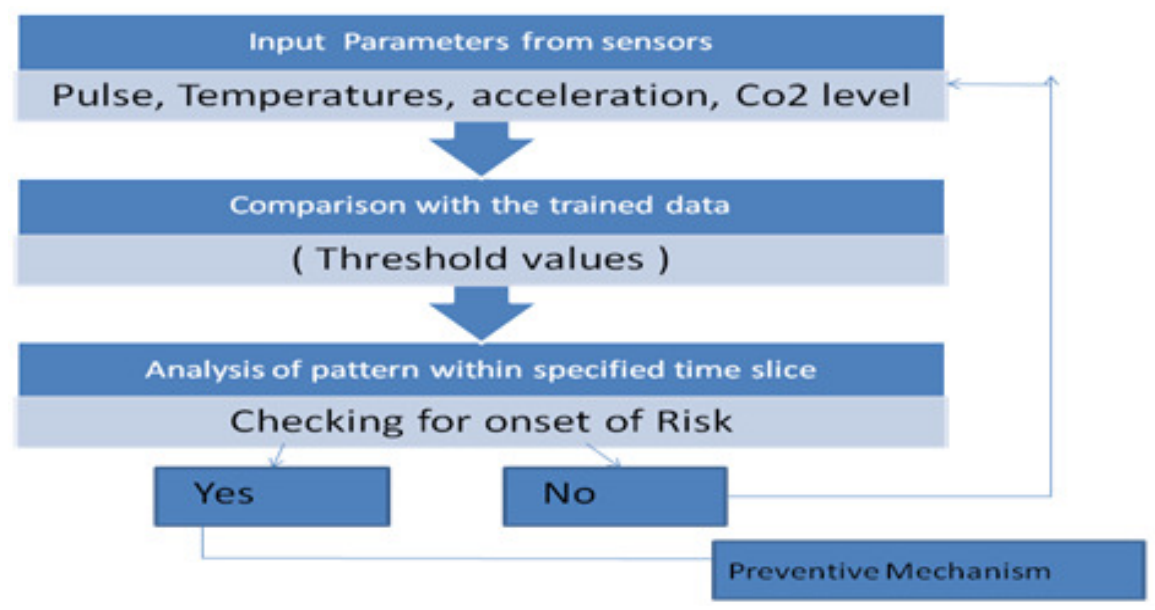

Fig. 2: Flow Diagram 
The flow diagram has been shown in fig2. This figure gives an pictorial view of the actual execution of the project. It elaborates that the real-time physiological parameters such as inner and outer temperature, pulse, $\mathrm{CO}_{2}$ level and accelerations will be monitored and analyzed with the help of training data for the desired time slice then the probability will be evaluated to see for the onset of the risk. With the increased value of probability, the preventive mechanism will be taken accordingly.

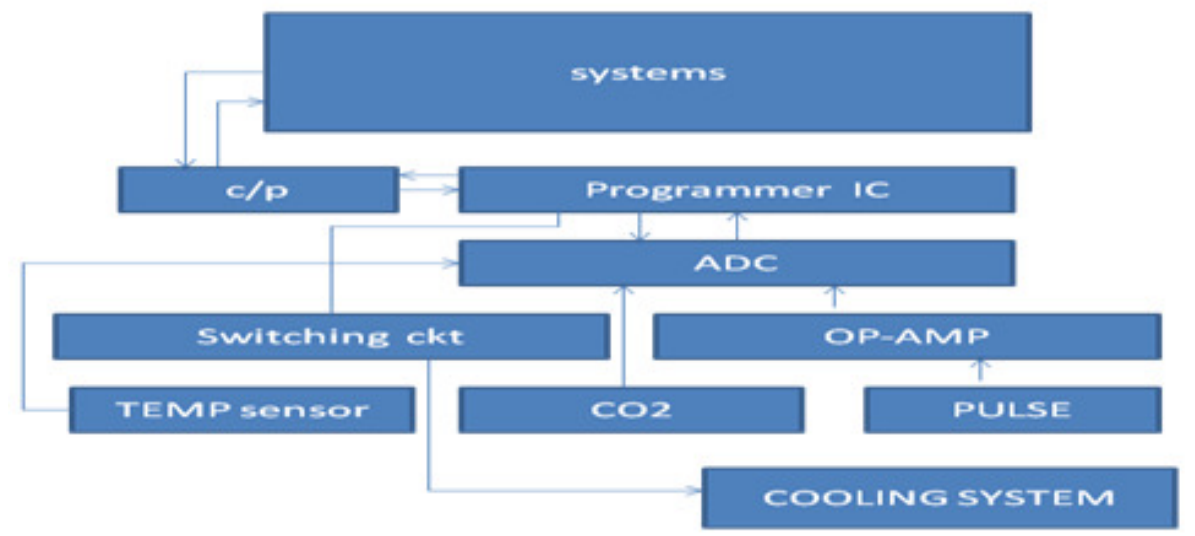

Fig3 : Experimental Setup

The parameter here considered are: inner temperature (inside the suit ), outer temperature (outside of the suit), number of accelerations, pulse rate and $\mathrm{CO}_{2}$ level. These parameters can be well used in order to predict if there is onset of the susceptible risk or not. The following block diagram shows the brief architectural model for gathering the real time parameter data as input the algorithm. Fig. 3 shows experimental setup that used for gathering the input data from the sensors. In the first module two parameters such as inner temperature and outer temperature were gathered and tested if a alert in the form of buzzer can be invoked if the threshold value that has been set extends. This was executed successfully. Fig. 4 shows testing of the first module. The testing of the first module was done successfully. The relays were used for switching between the temperatures: inner temperature and outer temperature. This is done to avoid number of analog to digital converter ICs. The circuit can be enhanced by many perspectives but since the focus is on the application of Bayesian Network Model author limited the involvement in hardware design since the aim with the hardware is to ultimately collect the sensor data and provide it to the system where the algorithm is residing for further processing.

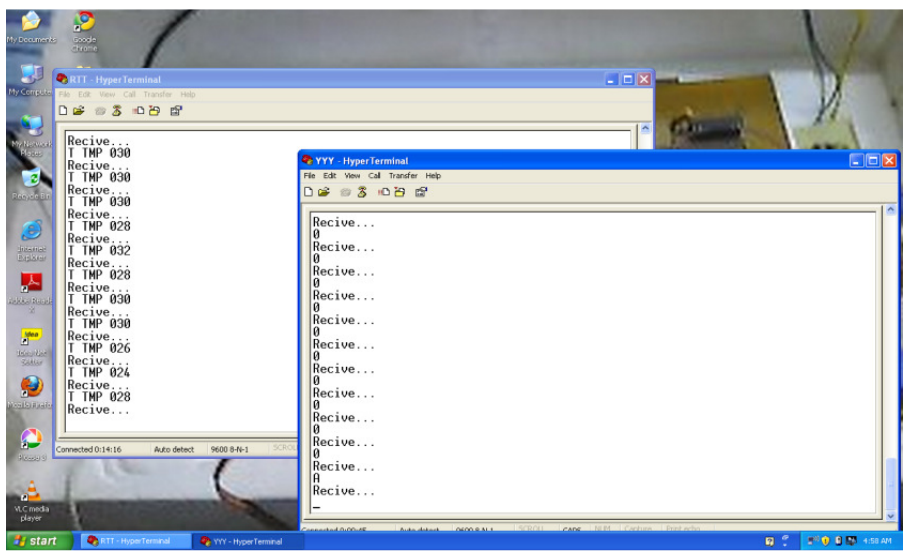

Fig.4: Testing of first module 
The testing on first module was done successfully. As a second module other parameters such as pulse, $\mathrm{CO}_{2}$ level, and accelerations were also considered and were also tested successfully as shown in fig 5 .

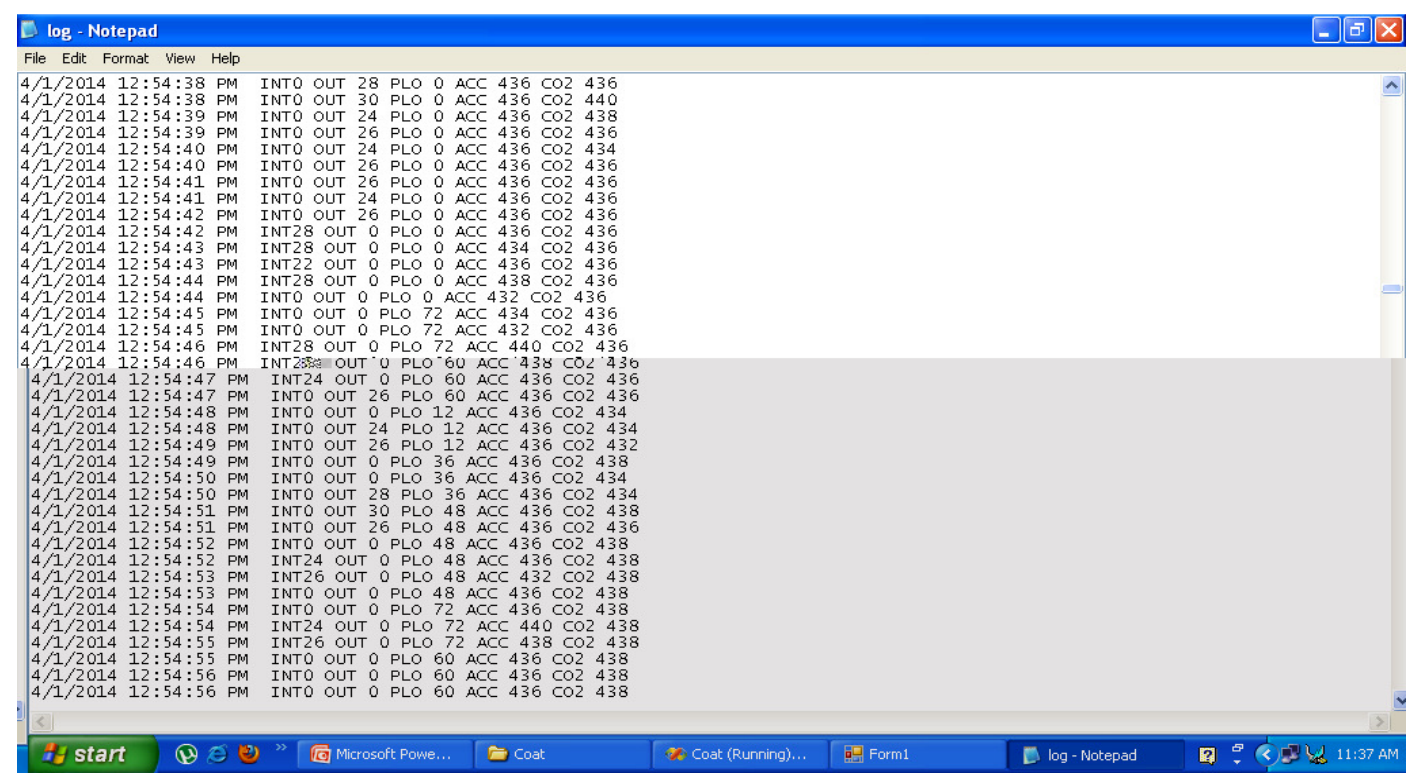

Fig. 5 : Testing result with inner/outer temperature, pulse, $\mathrm{CO}_{2}$ level and accelerations

In fig5. We can see that the readings are showing the value zero at some places this is because of the use relays and switching; though it has been managed that the switching time is as minimum as possible. The relays have been used to keep the hardware minimum yielding simplicity, also to reduce the cost. The use of relays allowed author to use single analog-to-digital convertor for the required no. of parameter sensors.

\section{USER INTERFACE}

The user interface of the system has been designed as simple as possible. It hardly includes complexity of the internal system so that operating can be made easy to the new user of the system.

The real-time parameter readings collected from the external hardware are also shown in the user interface to provide the user with information about what is happening with the suit-wearers body. The graphs are added to get the quick view of the changes in the parameters. For adding the new data to get more and more accurate results the options are provided in the user interface. At the beginning it was avoided to add a large data base regarding the training data in order to minimize the comparisons so as to minimize the execution time. 


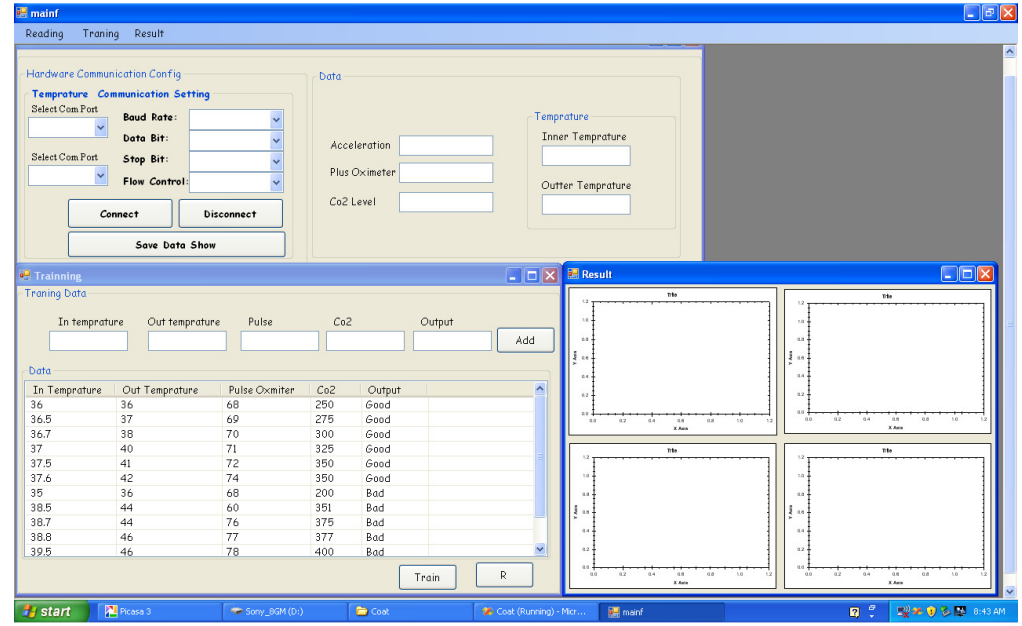

Fig 4: User Interface

\section{IMPLEMENTATION}

The project is designed in such a way that it can be viewed as an embedded one. The operative can carry the kit with him along with wired connection to the system ( laptop ) kept in the shoulder bag of the operative. The system can be used as a whole or can used as a single unit; only a $\mathrm{CO}_{2}$ monitoring system along with other parameters or as a whole with $\mathrm{CO}_{2}$ monitoring as well as with the suit specifically for temperature monitoring. The following fig. 5 gives of both the system. This gives the user to use the system according to the requirement of various environments.
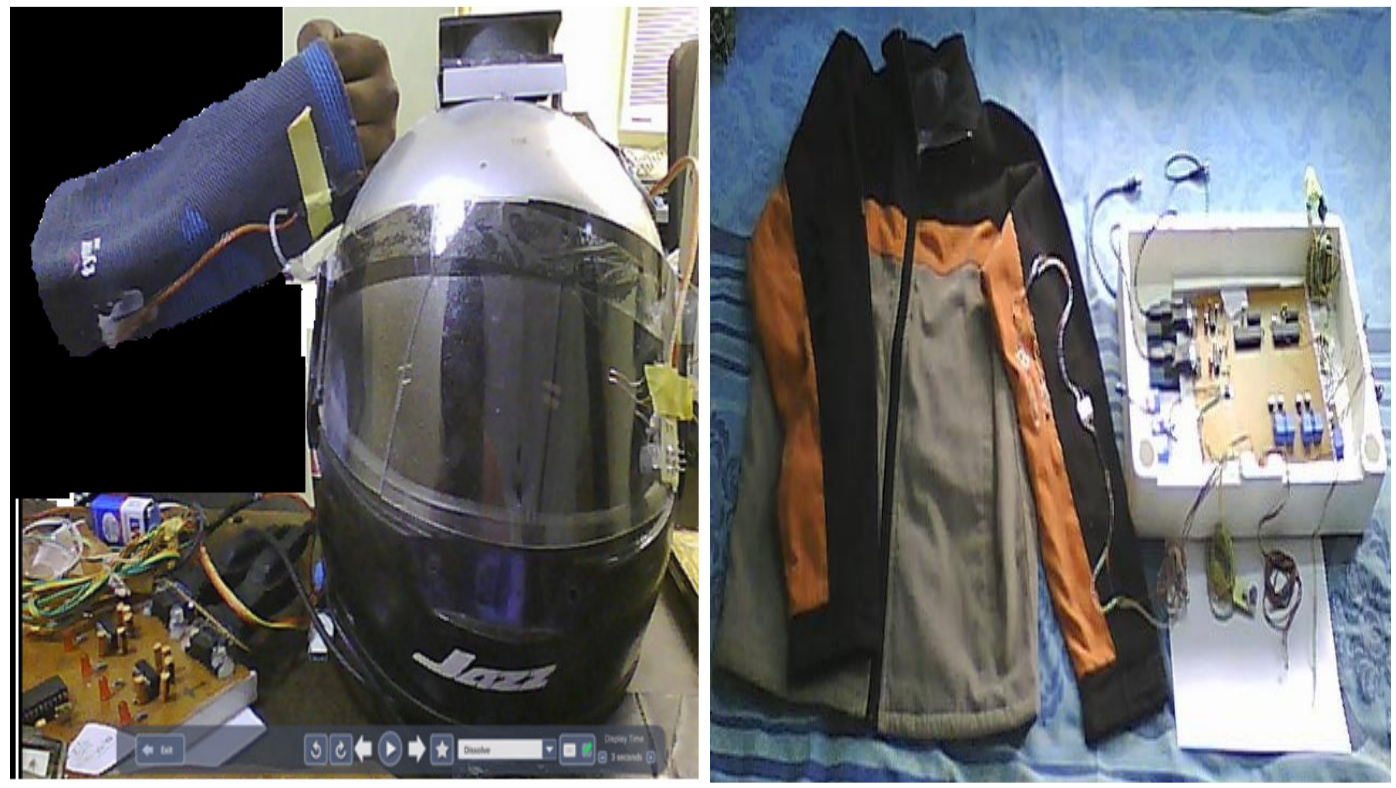

Fig 5. System can be used as a single unit or as a whole along with temperature monitoring. 


\subsection{Matching Technique}

$\mathrm{T}_{\text {sk,t+1 }}$ : Be the event when $\mathrm{T}_{\mathrm{sk}, t+1}>36^{0} \mathrm{C}$

Then Aim :

$\mathrm{P}\left(\mathrm{T}_{\mathrm{sk}, t+1} \mid \mathrm{S}\right)=\mathrm{P}(\mathrm{IT}) \mathrm{P}(\mathrm{OT}) \mathrm{P}(\mathrm{Pl}) \mathrm{P}(\mathrm{Ac}) \mathrm{P}\left(\mathrm{Co}_{2}\right)$

Normal : $\left\{\mathrm{It}, \mathrm{Ot}, \mathrm{P}, \mathrm{Ac}, \mathrm{Co}_{2}\right\}$

Where each of the parameter with probability value :

$1:$ Good

0 : Bad

As shown the condition Normal is treated as a tuple consisting of the probabilistic values of the parameters sensed. The parameters acquires the value as good or bad depending on their threshold values.The threshold values are taken both ways as minimum and maximum. If a particular parameter value exist between the minimum and maximum value then it will be treated as a good value i.e as a 1.If the parameter value either is below the minimum value or beyond the maximum value then it will be treated as an abnormal i.e. bad and numerically 0 .

Such various instances can be monitored at a regular time slice.A specific number of such patterns can be analyzed and evaluated to see for the probability that the temperature will reach the threshold value. Using the Bayesian Net [1] model for such type of prediction makes the modeling of the system simplified also it prevents from making complex calculations for evaluation and prediction as in the case of use of Kalman filtering approach [6].

\subsection{Selection of the Parameters}

The parameters in the system for monitoring are selected in such way that they are well-corelated and significantly contribute in the elevation of the body temperature. The skin temperature above $35^{\circ} \mathrm{C}$ reflects the inner temperature as mentioned in paper by Elena Gaura [1]. In this paper the author is not calculating the mean skin temperature to get the inner temperature as in [1], because pulse rate monitor has been used which specifies that if there is a increase in the pulse rate with this scenario then it is due to raise in inner temperature so increased pulse rate is a sign of increased inner temperature hence no need to calculate the inner temperature. The outer temperature parameter has been selected because the raise in outer temperature beyond the compensable rate increases the body inner temperature and the skin temperature. When body tries to cope with the raised temperature heart rate increases and to the breathing rate. If the helmet is wore on then $\mathrm{CO}_{2}$ volume inside the helmet will also increase and will require exhausting the $\mathrm{CO}_{2}$ to make breathing easy. Hence a fan is mounted on the helmet to exhaust the $\mathrm{CO} 2$ and keep the helmet environment normal. While wearing a protective suit [1] if an operative performs some physical activities, as he has to during mission, such as climbing, crawling, walking this also increased body temperature hence an acceleration sensor is also used to monitor the accelerations. If there is abnormality in more than two to three parameters for specific time period then the user will be prompted through the buzzer or the preventive mechanism will be made on automatically. In some specific cases such as fire-fighting scenario [1] if the external temperature is extremely high, in such situation the system can be equipped with some extra components such dry ice pack with circulatory pipes inside the suit as mentioned in paper [1]. On the same basis if the external environment outside the helmet is consisting of low oxygen volume then in such cases the 
operative can be provided with extra oxygen cylinder depending on which environment the system is going to be used.

\section{RESULT}

The system is evaluated with the real time parameters i.e. the readings were gathered with normal ranges as well as abnormal ranges while wearing an insulating material made suit as shown in fig. 5. The results obtained are shown as below in fig 6. With normal ranges of parameters obtained no action was taken out. As soon as the pattern of abnormal ranges were observed within the particular time slice the preventive mechanism, here it is fan, fan mounted on helmet, gets actuated automatically by the system as shown in fig. 7 .

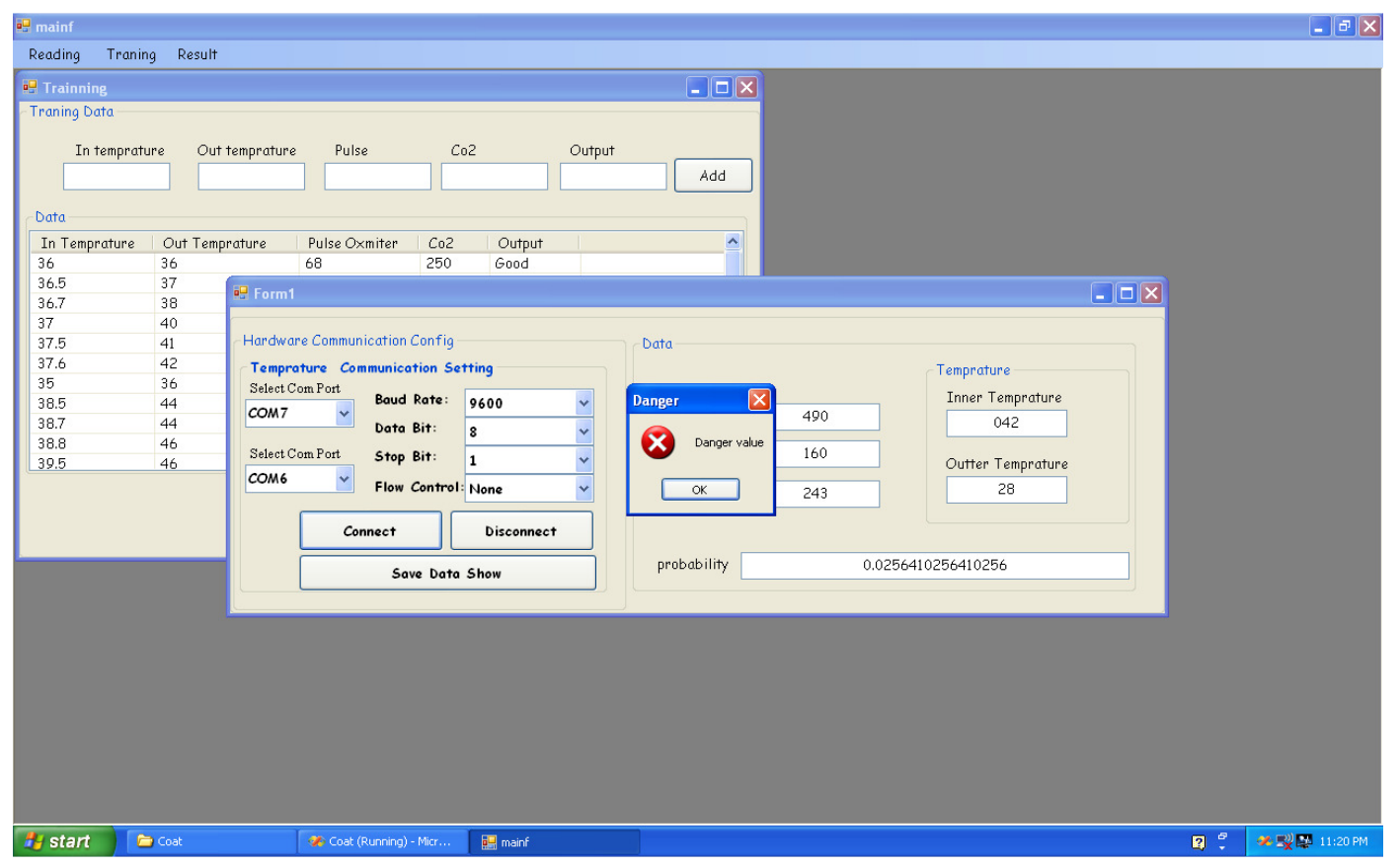

Fig. 6. With abnormality of the parameters the risk has been indicated.

In fig. 6 it can be observed that though the outer temperature is normal, there is increase in the accelerations leading to increase inner temperature indicated by the increase in pulse rate. Here it also be seen that with the increase in accelerations the increase in the $\mathrm{CO}_{2}$ level is also noted.

This is how the parameters are co-related and play significance role in the elevation of the body temperature. Fig. 7 shows actuation of the fan mounted on the helmet as a preventive mechanism of the system. To be mounted on the suit it needs a specific designed suit with the circulatory mechanism for the air inside the suit. 


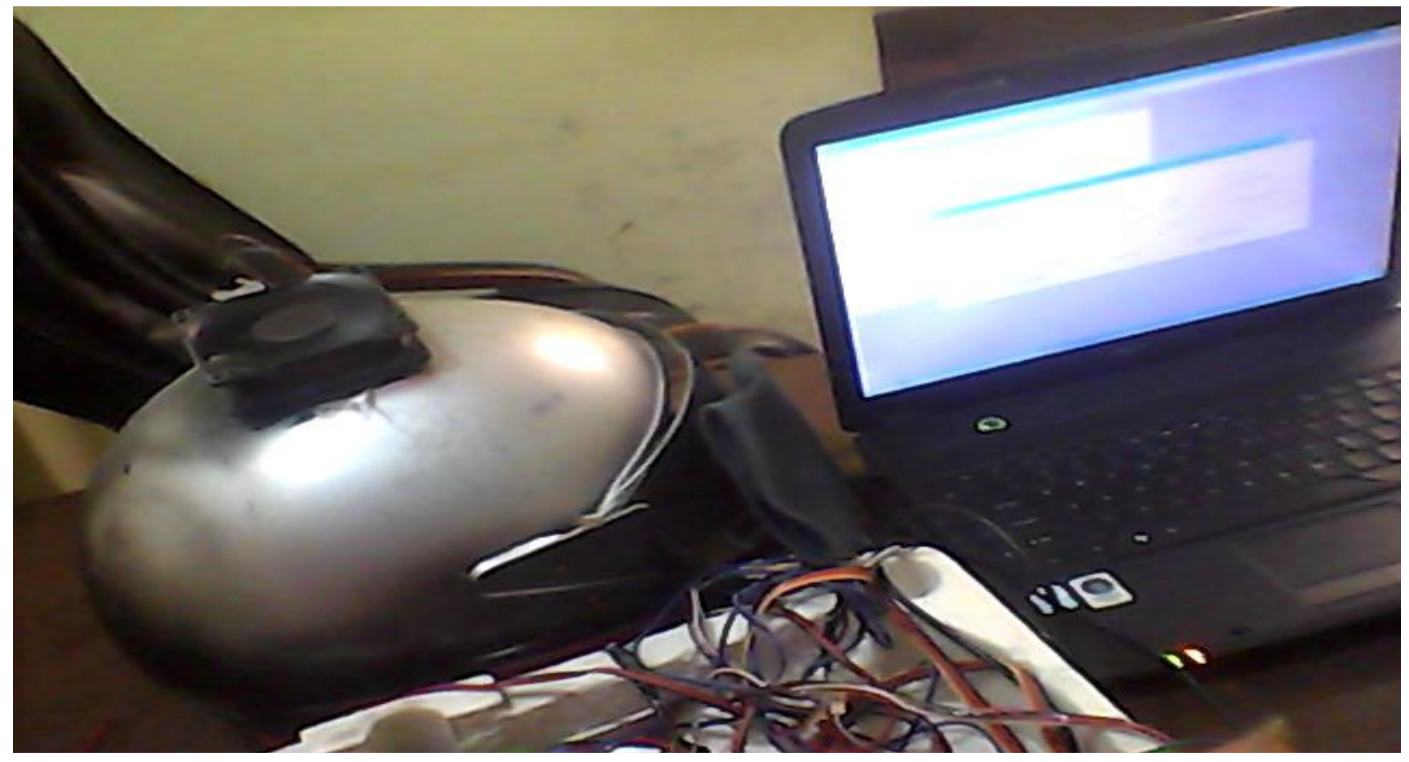

Fig. 7. As a preventive mechanism the fan has been switched on.

\section{CONCLUSION}

An autonomous system for UHS prediction has been established with low cost by using the Bayesian Net Model as a predictor [1]. The cost of the project is minimized by using relays that switches the use of analog-to-digital convertor between various sensor but yet not affect the quality of the project. The execution time is also minimized by not calculating the mean skin temperature to get the inner temperature which requires temperatures sensors to be placed at upper and lower limbs. Getting the accurate inner temperature is not the focus here instead if there is sign of increased inner temperature then hot to cope with it is the focus, hence the increased pulse rate is taken as a indicator of the increased inner temperature above compensable rate. Implementing this concept has reduced the calculations as well as use of temperature sensors leading to reduced time for execution of the predictor.

\section{FUTURE SCOPE}

Multiple environment suitability : Refinement of the model parameters may allow the same prediction mechanism to be employed in a variety of other applications. Such as Coal-mining, Fire-fighting operations, soldier training scenarios etc.

\section{ACKNOWLEDGEMENT}

The author would like to thank Prof. P. S. Mohod for his guidance and valuable comments.

\section{REFERENCES}

[1] Elena Gaura, Member, IEEE, John Kemp, and James Brusey, Member, IEEE, "Leveraging Knowledge From Physiological Data: On-Body Heat Stress Risk Prediction With Sensor Networks “, IEEE transactions on biomedical circuits and systems 2013.

[2] C. W. Mundt, K. N. Montgomery, U. E. Udoh, V. N. Barker, G. C. Thonier, A. M. Tellier, R. D. Ricks, and R. B. Darling, "A multiparameter wearable physiologic monitoring system for space and terrestrial applications," IEEE Trans. Inf. Technol. Biomed., vol. 9, no. 3, pp. 382-391, Sep. 2005. 
[3] R. Dilmaghani, H. Bobarshad, M. Ghavami, S. Choobkar, and C. Wolfe, "Wireless sensor networks for monitoring physiological signals of multiple patients," IEEE Trans. Biomed. Circuits Syst., vol. 5, no. 4, pp. 347-346, Aug. 2011.

[4] K. Li, S. Warren, and B. Natarajan, "Onboard tagging for real-time quality assessment of photoplethysmograms acquired by a wireless reflectance pulse oximeter," IEEE Trans. Biomed. Circuits Syst., vol. 6, no. 1, pp. 54-63, Feb. 2012.

[5] Y. Chuo, M. Marzencki, B. Hung, C. Jaggernauth, K. Tavakolian, P. Lin, and B. Kaminska, "Mechanically flexible wireless multisensor platform for human physical activity and vitals monitoring," IEEE Trans. Biomed. Circuits Syst., vol. 4, no. 5, pp. 281-294, Oct. 2010.

[6] E. Gaura, J. Brusey, J. Kemp, and C. D. Thake, "Increasing safety of bomb disposal missions: A body sensor network approach,” IEEE Trans. Syst., Man, Cybern. C, Applicat. Rev., vol. 39, no. 6, pp. 621636, Nov. 2009.

\section{AUTHORS}

\section{Kanchan M. Taiwade}

M. Tech student of Computer Science and Engineering. GHRIETW.

Nagpur University 2012-2014.

\section{Prof. Prakash S. Mohod}

Head of the Dept of Computer Science and Technology G.H. Raisoni College of Engg. and Technology for Women. Nagpur. 